\title{
CORONAL MAGNETIC FIELDS AND \\ ENERGETIC PARTICLES
}

\author{
GORDON NEWKIRK, JR. \\ High Altitude Observatory, National Center for Atmospheric Research*, Boulder, Colo., U.S.A.
}

\begin{abstract}
Magnetic fields in coronal and interplanetary space play a critical role in the propagation and storage of energetic particles from the flare site to $1 \mathrm{AU}$. The current investigation is designed to determine if the detailed configuration of the coronal magnetic fields below $2.5 R_{\odot}$ has a measurable influence on the escape of particles to $1 \mathrm{AU}$, can account for the observed broad longitudinal distribution of proton flares, and can provide a realistic site for storage of energetic particles.
\end{abstract}
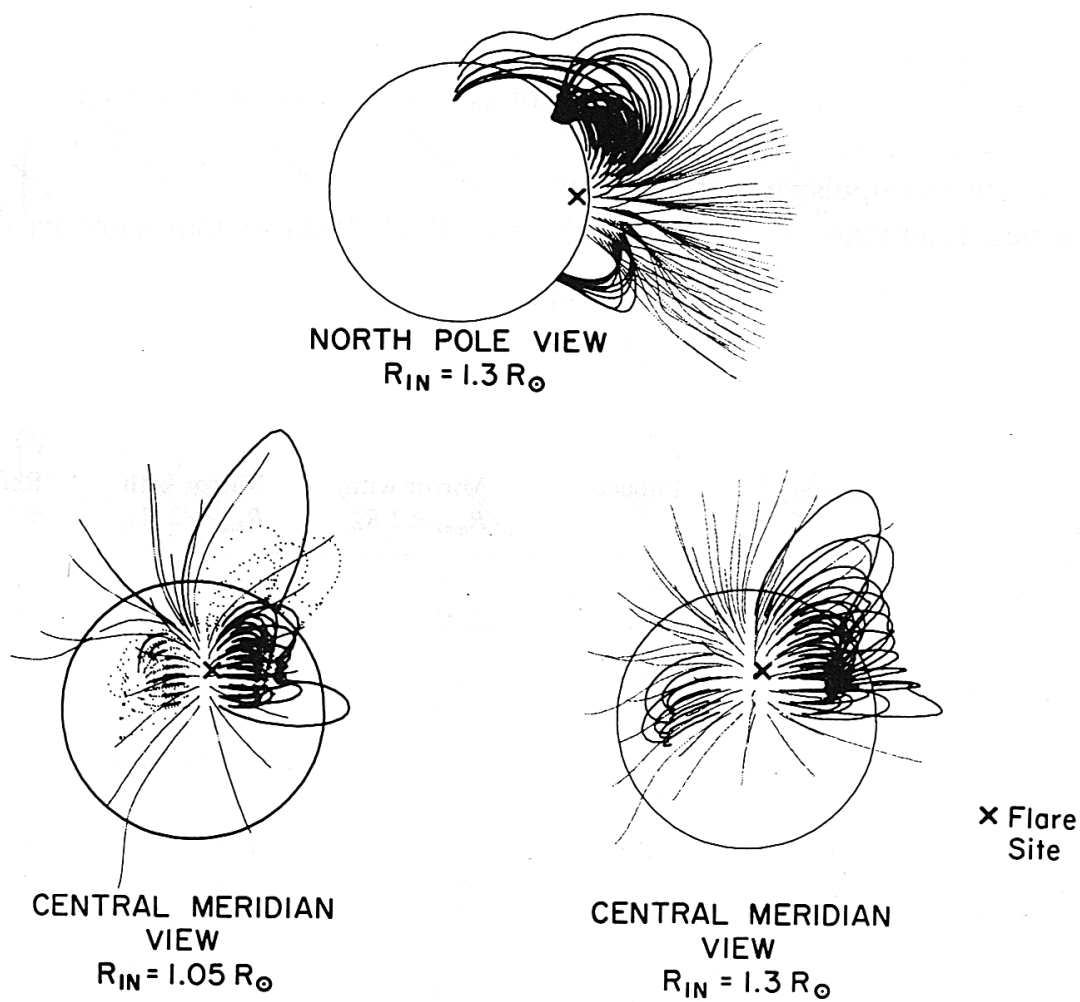

CENTRAL MERIDIAN
VIEW
$R_{I N}=1.3 R_{\odot}$

Fig. 1. A typical example of the trajectories of particles originating at a $36^{\circ} \times 36^{\circ}$ injection surface centered on a flare $(x)$ for two different values of $R_{\text {in }}$. Mirroring particle trajectories appear as heavy solid lines; escaping trajectories, as light solid lines; and impacting trajectories, as dotted lines. The particular event displayed is the long duration proton flare of 20 April 1971.

* The National Center for Atmospheric Research is sponsored by the National Science Foundation.

** In R. Ramaty and R. G. Stone (eds.), Proc. of Symposium on High Energy Phenomena on the Sun. 
Particles are followed in the ambient magnetic field from their injection in the mirroring condition at a surface above a flare until they either:

(1) impact on the photosphere;

(2) escape into interplanetary space;

(3) mirror at some distant location in the corona.

We use the guiding center approximation but neglect drifts, pitch angle diffusion, and scattering from magnetic inhomogeneities. Energy loss by Coulomb collisions is included for a model corona. The ambient magnetic fields are calculated using a potential model incorporating Mt. Wilson photospheric magnetic data. A typical example of such field line calculations for an injection surface placed at $1.3 R_{\odot}$ covering a sector $36^{\circ} \times 36^{\circ}$ centered on the flare appears in Figure 1 .

The calculations are applied to 46 flares of reported importance $>2+$ which occurred in the period 1959-1971 at times when good magnetic data were available. To minimize the influence of any systematic effect of the spiral interplanetary field, attention was concentrated on the 27 western hemisphere flares. These included:

(1) 14 flares without proton events;

(2) 6 flares with long duration (lifetimes in excess of 3 days at $100 \mathrm{MeV}$ ) or with recurrent proton events;

(3) 7 flares with impulsive proton events.

A statistical examination of these calculations (Table I) shows that many particles

TABLE I

Probability of injected particles having a particular fate

$\left(36^{\circ}\right.$ Injection sector at $\left.1.3 R_{\odot}\right)$

\begin{tabular}{|c|c|c|c|c|c|}
\hline \multirow[t]{2}{*}{ (1) } & (2) & (3) & (4) & (5) & (6) \\
\hline & Escape & Impact & $\begin{array}{l}\text { Mirror with } \\
R_{\max }<2 R_{\odot}\end{array}$ & $\begin{array}{l}\text { Mirror with } \\
R_{\max }>2 R_{\odot}\end{array}$ & $\begin{array}{l}\text { Remain } \\
\text { at Sun }\end{array}$ \\
\hline $\begin{array}{l}\text { All flares }>2+ \\
\quad(46)\end{array}$ & $\begin{array}{r}0.28 \\
531\end{array}$ & $\begin{array}{r}0.002 \\
3\end{array}$ & $\begin{array}{l}0.68 \\
1287\end{array}$ & $\begin{array}{r}0.04 \\
79\end{array}$ & $\begin{array}{l}0.72 \\
1369\end{array}$ \\
\hline $\begin{array}{l}\text { Western hemisphere } \\
\text { flares }>2+\text {, } \\
\text { non-proton } \\
\quad(14)\end{array}$ & $\begin{array}{r}0.16 \\
222\end{array}$ & $\begin{array}{r}0.001 \\
1\end{array}$ & $\begin{array}{l}0.80 \\
1127\end{array}$ & $\begin{array}{r}0.04 \\
50\end{array}$ & $\begin{array}{l}0.84 \\
1178\end{array}$ \\
\hline $\begin{array}{l}\text { Western hemisphere } \\
\text { flares }>2+\text {, } \\
\text { long duration proton } \\
\text { (6) }\end{array}$ & $\begin{array}{r}0.24 \\
143\end{array}$ & $\begin{array}{r}0.003 \\
2\end{array}$ & $\begin{array}{r}0.74 \\
441\end{array}$ & $\begin{array}{r}0.02 \\
14\end{array}$ & $\begin{array}{r}0.76 \\
457\end{array}$ \\
\hline $\begin{array}{l}\text { Western hemisphere } \\
\text { flares }>2+\text {, } \\
\text { impulsive proton } \\
\quad \text { (7) }\end{array}$ & $\begin{array}{r}0.39 \\
276\end{array}$ & $\begin{array}{r}0.004 \\
3\end{array}$ & $\begin{array}{r}0.58 \\
405\end{array}$ & $\begin{array}{r}0.02 \\
16\end{array}$ & $\begin{array}{r}0.61 \\
424\end{array}$ \\
\hline
\end{tabular}

Note: The division between impulsive and long duration proton flares is taken as a reported lifetime of 3 days for $100 \mathrm{MeV}$ protons. Number of flares appear in parenthesis while number of field lines appears in italics. 
$(\sim 60 \%)$ find themselves initially mirroring in closed magnetic arches, that a smaller fraction (16-40\%) escape immediately, and that a still smaller fraction (1-15\%) impact onto the photosphere. However, only a small number $(\sim 4 \%)$ of the injected particles would find themselves mirroring on field lines which extended above $2.0 R_{\odot}$. Differences in the ambient magnetic field configurations between proton and nonproton flares are also examined with the conclusion that proton flares have significantly more open field lines emerging from the injection surface than do flares of the

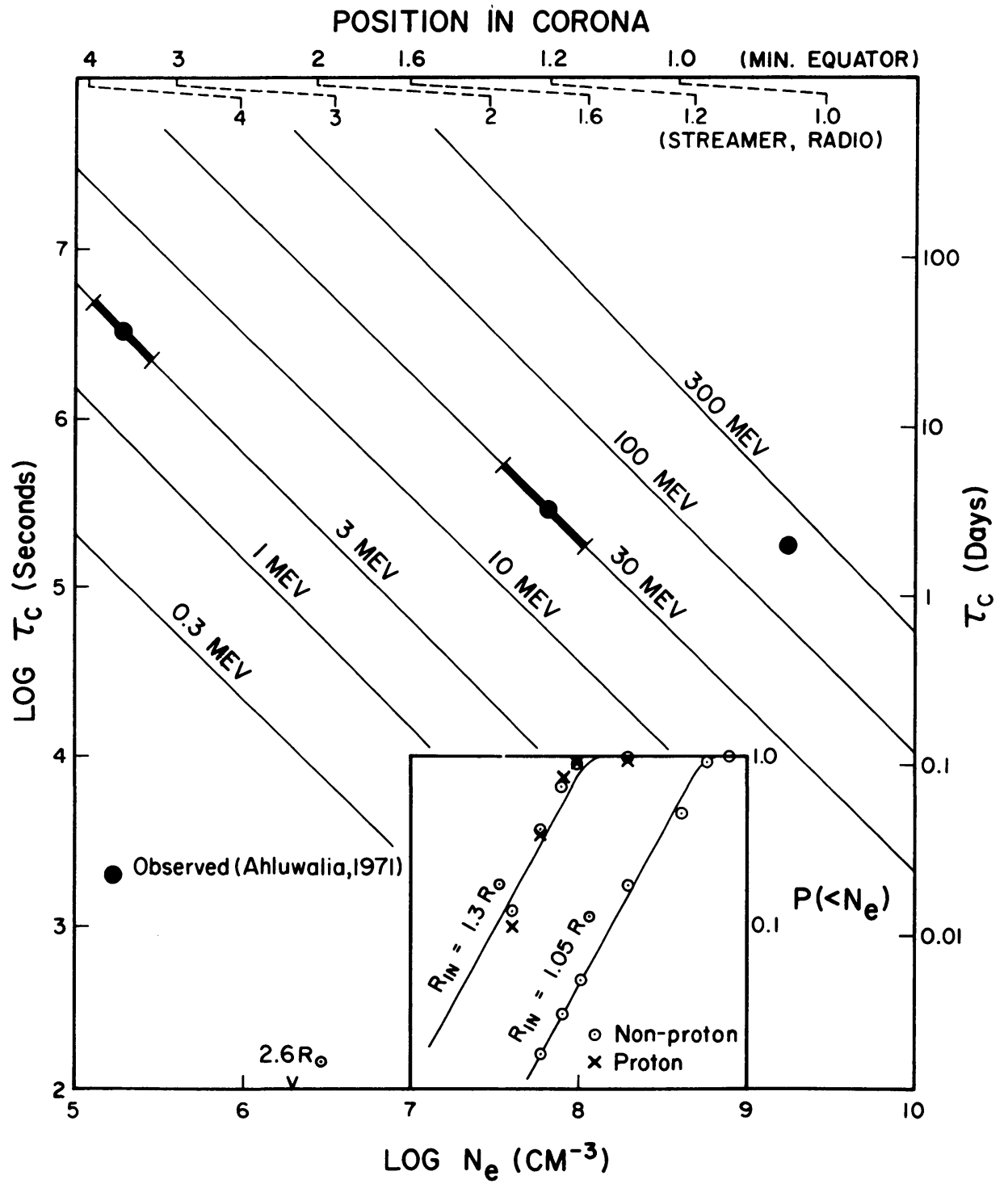

Fig. 2. The lifetimes of protons of various energies moving in a completely ionized corona of density $N_{e}$ together with the equivalent position in the corona for each density. The inset shows the probability that temporarily trapped particles will encounter an average density less than $N_{e}$. 
same importance which do not produce protons at $1 \mathrm{AU}$. We conclude that the ambient field configuration has a detectable influence on whether or not protons escape from a given flare.

Comparing the calculation of the average spread in longitude of field lines opened by the solar wind at $2.5 R_{\odot}$ with observations of proton and electrons at $1 \mathrm{AU}$, we conclude that the present model cannot account for the observed longitude distribution of energetic particles.

The mean electron density encountered by particles mirroring in the magnetic field permits us to determine the probability that particles injected from the flare regions will encounter a mean electron density smaller than any given value. Mean electron densities smaller than $\sim 3 \times 10^{7} \mathrm{~cm}^{-3}$ occur for less than $10 \%$ of the mirroring particles (Figure 2). This implies that storage of $10 \mathrm{MeV}$ particles for longer than 1 day and for $3 \mathrm{MeV}$ particles for longer than a few hours is extremely unlikely. Thus, particle storage in high magnetic arches cannot account for the long duration (1-50 days) of particles with energies $\approx 10 \mathrm{MeV}$ at $1 \mathrm{AU}$. Such particles must be generated by a continuously-operating acceleration mechanism.

\section{DISCUSSION}

Duncan: Particles injected at a position where the field strength is $B$ cannot mirror until they encounter a field strength equal to or greater than $B$. Hence, in a symmetrical loop most of them will plunge back into the chromosphere. If only a negligible number impact, it implies that the magnetic field strength in the vicinity of the flare is less than that on other parts of the chromosphere. Is this what you are inferring?

Newkirk: No. This calculation assumes that particles are injected in the mirroring condition, and thus those particles which would strike the chromosphere immediately below the injection surface are neglected. The fraction of isotropically injected particles which would impact would be 5 to 10 times higher than I have indicated.

Wentzel: This situation inevitably leads to velocity anisotropics; these cause various plasma instabilities; the resulting waves scatter the particles and greatly increase the loss rate. Depending on particle densities, time scales of minutes are typical.

Newkirk: I agree. These calculations represent an upper limit to the time particles can be stored.

Smerd: The problem may be related to that of meter-wave type I storms for similar periods. If the type I-radiating electrons are accelerated intermittently throughout the storms to $\mathrm{keV}$ in a Fermi-like manner by MHD waves in a closed magnetic loop, the corresponding protons would reach energies of $\mathrm{MeV}$. These protons could account for the observed sub-relativistic proton storms when they diffuse out of the closed-field region. 